\title{
Análise do perfil das cirurgias cardiovasculares do Hospital Universitário Walter Cantídio
}

\section{Analysis of the profile of cardiovascular surgeries at Universitary Hospital Walter Cantídio}

Matheus Duarte Pimentel ${ }^{1}$. Caroline Cleophas Lobo Leite ${ }^{1}$. Carolina Oliveira Sousa ${ }^{1}$. Isadora Lopes Bessa ${ }^{1}$. Gladson Fernandes Vieira². Heraldo Guedis Lobo Filho². José Glauco Lobo Filho².

1 Universidade Federal do Ceará (UFC), Fortaleza, Ceará, Brasil. 2 Hospital Universitário Walter Cantídio (HUWC), Universidade Federal do Ceará (UFC), Fortaleza, Ceará, Brasil.

\section{RESUMO}

Objetivo: analisar o perfil das cirurgias cardiovasculares (CCV) realizadas no Hospital Universitário Walter Cantídio (HUWC) de 2009 a 2016, comparando este com o verificado no restante do Brasil. Material e Métodos: neste estudo observacional e retrospectivo, analisou-se em banco de dados os tipos de CCV no período citado, sendo estes agrupados por tipo e ano. Análise local foi então comparada com dados do DATASUS. Resultados: realizou-se 680 procedimentos no período analisado. Destes, 392 $(57,72 \pm 9,4 \%)$ cirurgias de revascularização do miocárdio (CRM), $199(29,28 \pm 6,16 \%)$ cirurgias orovalvares, $36(5,54 \pm 3,09 \%)$ CRM combinada a procedimentos orovalvares, $21(3,01 \pm 2,45 \%)$ correções de cardiopatias congênitas, e 33 (4,62 $\pm 2,70 \%)$ outros procedimentos. Nacionalmente, realizou-se, no período analisado, 354.129 procedimentos em CCV, dos quais 181.570 $(51,27 \pm 1,02 \%)$ foram CRM, $85.392(24,11 \pm 0,25 \%)$ cirurgias orovalvares, $16.677(4,71 \pm 0,35 \%)$ CRM associadas a troca e/ ou plastia valvar, $39.086(11,04 \pm 1,29 \%)$ correções de cardiopatias congênitas e $31.404(8,87 \pm 0,26 \%)$ outros procedimentos. Conclusão: perfil das CCV do HUWC ilustra que as CRM são o procedimento mais realizado, seguido das cirurgias orovalvares, em consonância com o verificado nacionalmente. Análise contínua do banco de dados local amplia perspectivas, permitindo melhores planejamentos e resultados.

Palavras-chave: Procedimentos cirúrgicos cardiovasculares. Base de dados. Sistema Único de Saúde.

\section{ABSTRACT}

Objective: To analyse the profile of cardiovascular surgeries (CVS) performed at Walter Cantídio University Hospital (HUWC) from 2009 to 2016, comparing it with that observed in the remainder of Brazil. Methods: In this observational and retrospective study, types of CCV were analysed in our database, in the mentioned period, these being grouped by type and year. Local analysis was then compared with data from DATASUS. Results: 680 procedures were performed in the analysed period. Of these, 392 $(57.72 \pm 9.4 \%)$ coronary artery bypass grafting (CABG), $199(29.28 \pm 6.16 \%)$ valvar surgeries, $36(5.54 \pm 3.09 \%)$ combined CABG and valvar surgeries, $21(3.01 \pm 2.45 \%)$ corrections for congenital heart defects, and $33(4.62 \pm 2.70 \%)$ other procedures. At the national level, 354,129 CVS procedures were performed, 181,570 (51.27 $\pm 1.02 \%)$ CABG, 85,392 (24.11 $\pm 0.25 \%)$ valvar surgeries, 16,677 (4.71 $\pm 0.35 \%)$ CABG combined with valvar surgery, 39,086 (11.04 $\pm 1.29 \%)$ corrections of congenital heart defects and 31,404 (8.87 $\pm 0.26 \%)$ other procedures. Conclusion: Profile of CVS of HUWC illustrates that CABG is the most performed procedure, followed by valvar surgeries, in line with nationally verified. Continuous analysis of the local database broadens perspectives, enabling improvement of planning and results.

Keywords: Cardiovascular surgical procedures. Database. Unified Health System.

Autor correspondente: Matheus Duarte Pimentel, Rua Suíça, 120, Maraponga, Fortaleza, Ceará. CEP: 60711-035.Telefone: +55 85 98646-4115. E-mail: pimentelmd@outlook.com

Conflito de interesses: Não há qualquer conflito de interesses por parte de qualquer um dos autores.

Recebido em: 04 Mar 2018; Revisado em: 29 Jul 2018; Aceito em: 29 Jul 2018. 


\section{INTRODUÇÃO}

No território brasileiro, o Sistema Único de Saúde (SUS) tem importância fundamental no planejamento e execução das políticas de saúde nacionais, uma vez que por seus princípios de universalidade, equidade e integralidade, visa garantir o direito e o acesso à saúde para toda a população brasileira. ${ }^{1-4}$ Apenas em 2016, este sistema de saúde foi responsável por 11.280.434 internações, das quais 470.383 ocorreram no estado do Ceará (4,16\%), dentro de uma população de 8.963.663 habitantes neste estado, ${ }^{2}$ dados estes que ilustram sua relevância, devendo-se destacar ainda que isso ocorre em uma população onde 1.252 .586 de indivíduos $(13,97 \%$ da população) possuem planos de saúde complementar. ${ }^{3-7}$

A cirurgia cardiovascular, por sua vez, constitui-se como área de destacada relevância no tratamento das afecções do sistema circulatório. $^{6-11} \mathrm{O}$ crescimento desta especialidade ao longo do século XX permitiu que, atualmente, procedimentos cada vez mais complexos pudessem ser realizados, aumentando a abrangência das abordagens terapêuticas, promovendo melhora no prognóstico dos pacientes, e permitindo que cada vez mais cirurgias deste tipo pudessem ser realizadas dentro do SUS. ${ }^{8-12}$

Atualmente, uma vez que os controles de gastos em procedimentos hospitalares de elevada complexidade mostram-se cada vez mais rigorosos, e que as expectativas acerca dos parâmetros de qualidade de assistência médica elevam-se gradativamente com o avanço tecnológico, uma avaliação sistemática da qualidade dos serviços prestados pelas equipes de cirurgia cardiovascular torna-se cada vez mais relevante. $4,6,7,9,10,11,13-19$

Desta forma, a criação de um banco de dados que contemple as cirurgias cardiovasculares realizadas no Hospital Universitário Walter Cantídio (HUWC) adquire relevância ao permitir a análise não apenas dos indicadores de desempenho do serviço, mas também do número de procedimentos realizados e de uma melhor estratificação de risco dos pacientes operados e dos desfechos dos procedimentos aos quais estes indivíduos foram submetidos. Além disto, permite reflexão e melhor planejamento acerca de como abordar da melhor forma a população de pacientes que necessita submeter-se a cirurgias cardiovasculares no hospital terciário em questão.

O objetivo deste trabalho é avaliar, através da revisão do banco de dados do HUWC, os tipos de procedimentos mais realizados em cirurgia cardiovascular entre os anos de 2009 e 2016, comparando o perfil deste centro aos dados do Sistema de Informações Hospitalares (SIH) do Sistema Único de Saúde (SUS), no mesmo período.

\section{MATERIAL E MÉTODOS}

Este estudo foi aprovado pelo Comitê de Ética em Pesquisa do HUWC sob o parecer de número 1.334.044. Os dados relativos ao HUWC foram obtidos utilizando o banco de dados do Serviço de Cirurgia Cardiovascular desta instituição, com a coleta das informações referentes aos procedimentos cirúrgicos realizados, sendo analisados os números e os tipos de cirurgias realizadas a cada ano. Os dados nacionais foram obtidos com uso do Sistema de Informações Hospitalares (SIH) do SUS, dentro de sua base de dados, o DATASUS. ${ }^{2}$ As informações de ambos os bancos de dados são referentes ao período entre aos anos de 2009 e 2016, por este constituir-se como o mais recente no qual os dados anuais estavam compilados no momento da realização deste estudo.

Os procedimentos realizados no HUWC incluíram: cirurgia de revascularização do miocárdio (CRM) isoladas, cirurgias para tratamento de afecções orovalvares, englobandose trocas valvares e plastias valvares, CRM associada a cirurgia orovalvar, cirurgia para correção de cardiopatias congênitas, em adultos e crianças. Foram agrupados como outros procedimentos cardiorrafia, cardiotomia para retirada de corpo estranho, pericardiectomia, drenagem com biópsia de pericárdio, exérese de cisto pericárdico, infartectomia, anastomose sistêmico-pulmonar com uso de circulação extracorpórea (CEC) e cirurgias para correção de aneurismas e dissecções da aorta torácica devido à infrequência de realização destes procedimentos no HUWC no período analisado.

Desta forma, após análise do número de operações realizadas no HUWC de cada grupo a ser estudado, buscou-se no SIH/ DATASUS, o número de Autorizações de Internação Hospitalar (AIH) do subgrupo de procedimentos "040601 - Cirurgia Cardiovascular”, selecionando-se dentro desta seção apenas os procedimentos em cirurgia cardiovascular realizados no HUWC, excluindo-se da seleção, portanto, os procedimentos em hemodinâmica, em cirurgia vascular periférica e em estimulação cardíaca artificial. Com as informações do banco de dados do HUWC e do DATASUS compiladas, procedeu-se à análise comparativa entre os dados.

\section{RESULTADOS}

No período analisado, foram realizadas 680 cirurgias cardiovasculares no HUWC. Destas, 392 foram CRM, 199 cirurgias para tratamento de afecções orovalvares, 36 CRM associadas a troca e/ou plastia valvar, 21 cirurgias para correção de cardiopatias congênitas e 33 constituíram outros tipos de procedimentos não agrupáveis nas seções anteriores. A Tabela 1 ilustra a distribuição quantitativa de cada grupo de procedimento avaliado anualmente.

No que diz respeito ao Brasil, segundo o SIH/DATASUS, no período analisado foram realizados 354.129 procedimentos em cirurgia cardiovascular, segundo os critérios utilizados neste estudo para agrupar os procedimentos. Destas, 181.570 corresponderam a CRM, 85.392 a cirurgias para tratamento de afecções orovalvares, 16.677 a CRM associadas a troca e/ou plastia valvar, 39.086 a cirurgias para correção de cardiopatias congênitas e 31.404 corresponderam a outros tipos de procedimentos não agrupáveis nas seções anteriores. A Tabela 2 ilustra a distribuição quantitativa de cada grupo de procedimento avaliado anualmente. Em análise qualitativa do perfil das cirurgias, os Gráficos 1 e 2, ilustram a evolução e distribuição percentual de cada grupo de procedimentos em cirurgia cardiovascular ao longo do período estudado. 
Tabela 1. Número de cirurgias cardiovasculares realizadas no Hospital Universitário Walter Cantídio, por grupo de procedimentos, entre 2009 e 2016.

\begin{tabular}{lccccccccc}
\hline Grupo de procedimentos & $\mathbf{2 0 0 9}$ & $\mathbf{2 0 1 0}$ & $\mathbf{2 0 1 1}$ & $\mathbf{2 0 1 2}$ & $\mathbf{2 0 1 3}$ & $\mathbf{2 0 1 4}$ & $\mathbf{2 0 1 5}$ & $\mathbf{2 0 1 6}$ & Total \\
\hline Correção de cardiopatia congênita & 7 & 2 & 4 & 2 & 2 & 1 & 0 & 3 & 21 \\
Cirurgias orovalvares isoladas & 32 & 13 & 24 & 25 & 25 & 23 & 23 & 34 & 199 \\
Cirurgia orovalvar associada a CRM & 6 & 2 & 2 & 8 & 6 & 3 & 6 & 3 & 36 \\
CRM isolada & 37 & 58 & 61 & 45 & 39 & 47 & 40 & 65 & 392 \\
Outros procedimentos & 2 & 1 & 6 & 0 & 5 & 4 & 3 & 10 & 31 \\
\hline
\end{tabular}

Dados expressos em valores absolutos. CRM: Cirurgia de Revascularização do Miocárdio.

Tabela 2. Número de cirurgias cardiovasculares realizadas no Brasil, segundo o SIH/DATASUS, por grupo de procedimentos, entre 2009 e 2016.

\begin{tabular}{lccccccccc}
\hline Grupo de procedimentos & $\mathbf{2 0 0 9}$ & $\mathbf{2 0 1 0}$ & $\mathbf{2 0 1 1}$ & $\mathbf{2 0 1 2}$ & $\mathbf{2 0 1 3}$ & $\mathbf{2 0 1 4}$ & $\mathbf{2 0 1 5}$ & $\mathbf{2 0 1 6}$ & Total \\
\hline Correção de cardiopatia congênita & 5829 & 5416 & 5325 & 4805 & 4645 & 4482 & 4378 & 4206 & 39086 \\
Cirurgias orovalvares isoladas & 10662 & 10266 & 10957 & 11165 & 10940 & 10848 & 10430 & 10124 & 85392 \\
$\begin{array}{l}\text { Cirurgia orovalvar associada a } \\
\text { CRM }\end{array}$ & 1874 & 1797 & 2146 & 2191 & 2183 & 2096 & 2136 & 2254 & 16677 \\
CRM isolada & 22077 & 21225 & 23187 & 23903 & 23368 & 23011 & 22559 & 22240 & 181570 \\
Outros procedimentos & 3997 & 3926 & 3954 & 3962 & 3904 & 3814 & 3914 & 3933 & 31404 \\
\hline
\end{tabular}

Dados expressos em valores absolutos. SIH/DATASUS: Sitema de Informações Hospitalares do Sistema Único de Saúde; CRM: Cirurgia de Revascularização do Miocárdio.

Gráfico 1. Distribuição e evolução, em percentuais relativos ao total das cirurgias cardiovasculares de cada ano, dos grupos de procedimentos realizados no Hospital Universitário Walter Cantídio, entre 2009 e 2016.

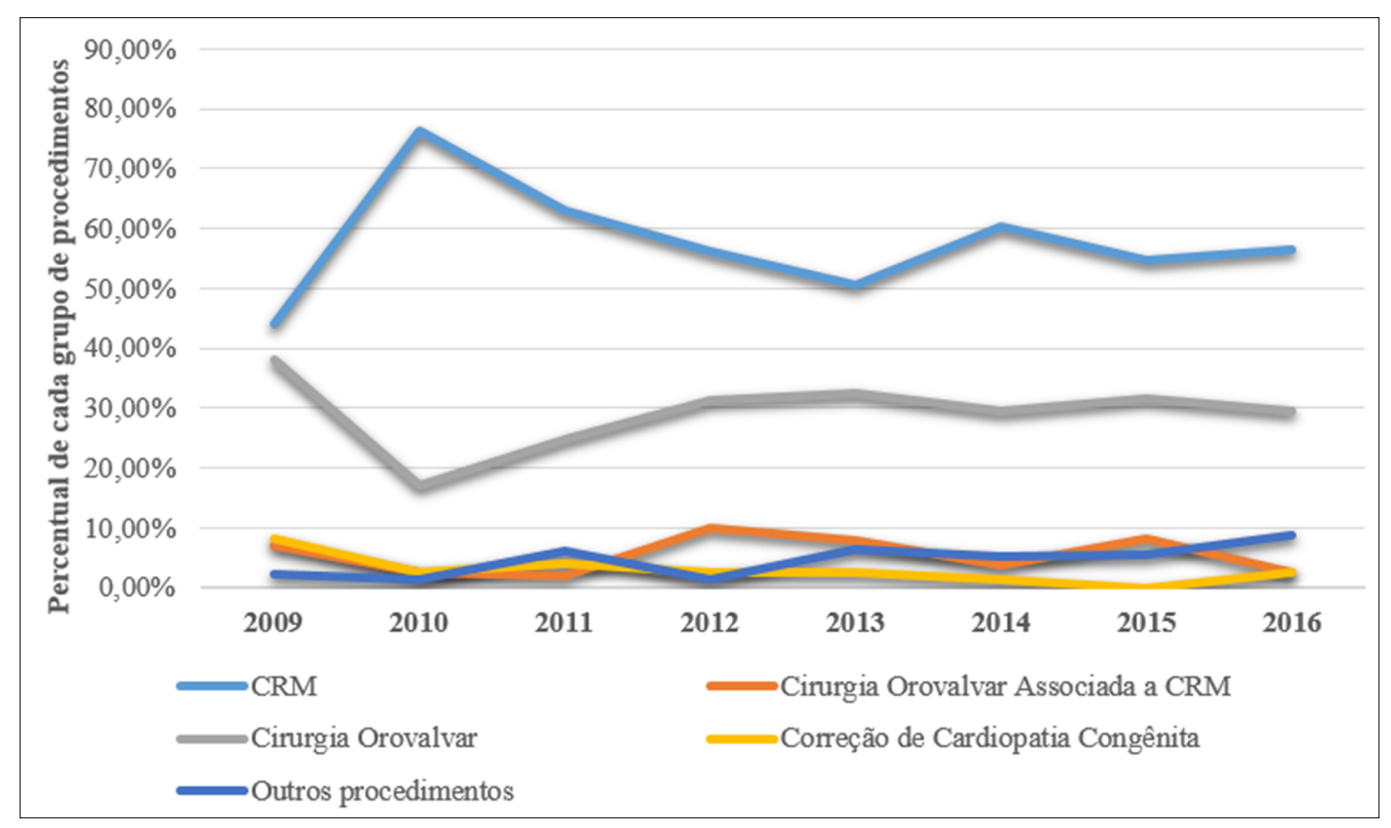

Dados expressos em valores percentuais relativos ao total de cirurgias realizadas em cada ano no HUWC. CRM: Cirurgia de Revascularização do Miocárdio. 
Consolidando-se os dados estudados entre 2009 e 2016, notase que no HUWC, a CRM correspondeu em média a 57,72 $\pm 9,4 \%$ dos procedimentos realizados, sendo seguida das cirurgias orovalvares isoladas, com 29,28 $\pm 6,16 \%$, cirurgias orovalvares associadas a CRM com 5,54 $\pm 3,09 \%$, cirurgias para correção de cardiopatias congênitas com 3,01 $\pm 2,45 \%$ e outros procedimentos em cirurgia cardiovascular com 4,62 $\pm 2,70 \%$.
No Brasil, a CRM correspondeu a 51,27 $\pm 1,02 \%$ das cirurgias cardiovasculares realizadas ao longo destes oito anos, sendo seguida das cirurgias orovalvares isoladas, $\operatorname{com} 24,11 \pm 0,25 \%$, cirurgias para correção de cardiopatias congênitas com 11,04 \pm $1,29 \%$, outros procedimentos em cirurgia cardiovascular com $8,87 \pm 0,26 \%$ e cirurgias orovalvares associadas a CRM com $4,71 \pm 0,35 \%$. O Gráfico 3 contempla a análise comparativa entre o HUWC e o Brasil no que diz respeito ao perfil das cirurgias cardiovasculares realizadas.

Gráfico 2. Distribuição e evolução, em percentuais relativos ao total das cirurgias cardiovasculares de cada ano, dos grupos de procedimentos realizados no Brasil, segundo o SIH/DATASUS, entre 2009 e 2016.

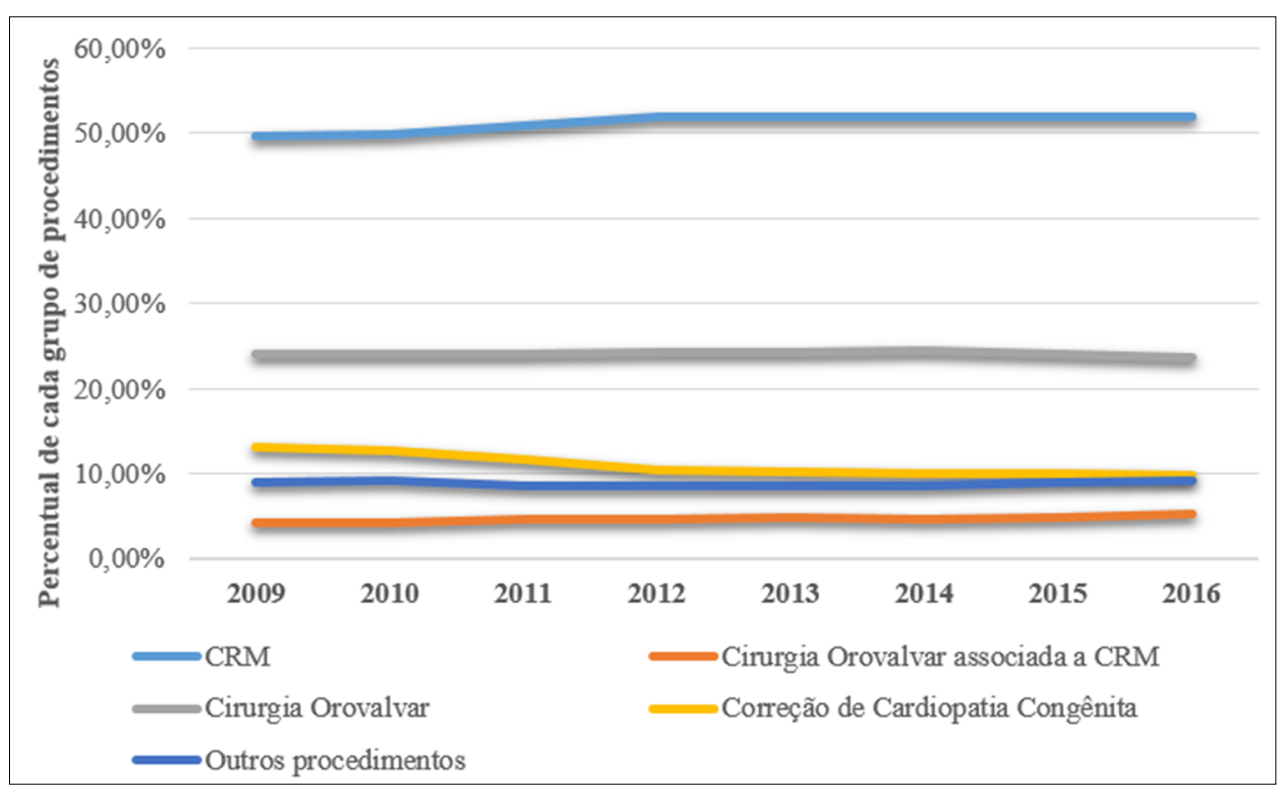

Dados expressos em valores percentuais relativos ao total de cirurgias realizadas em cada ano no HUWC. CRM: Cirurgia de Revascularização do Miocárdio.

Gráfico 3. Comparação do perfil das cirurgias no Hospital Universitário Walter Cantídio e no Brasil, segundo o SIH/DATASUS, entre 2009 e 2016.

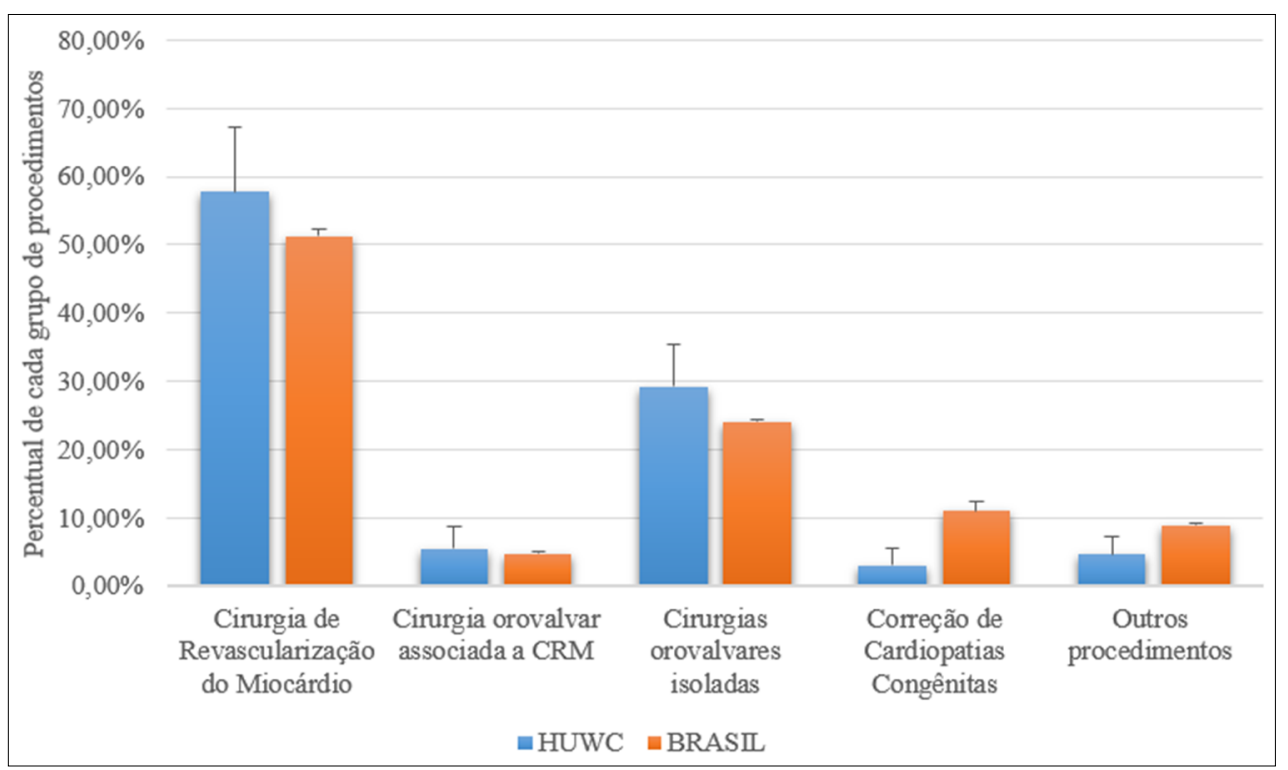

Dados expressos em valores percentuais relativos ao total de cirurgias realizadas em cada ano no HUWC. CRM: Cirurgia de Revascularização do Miocárdio. 


\section{DISCUSSÃO}

As patologias cardiovasculares são causa relevante de morbimortalidade no país. Nesse contexto, o controle de qualidade das cirurgias cardiovasculares deve ser prioridade nacional dado que o nível de qualidade do serviço é inversamente proporcional a sua mortalidade. Desse modo, a avaliação dos dados desses serviços se configura como importante instrumento para este fim. ${ }^{9}$

Os fundamentos do controle de qualidade em serviços hospitalares englobam fatores como: conhecimento dos procedimentos realizadas, avaliação criteriosa dos êxitos e das áreas a serem aprimorados, comparação constante com resultados de outros serviços, e compromisso com a publicização dos resultados..$^{20}$

Dessa forma, o resultado final de um procedimento cirúrgico é interdependente, em grande parte, de três fatores: equipe cirúrgica; estrutura hospitalar - que interfere em fatores como a agilidade na realização de exames, e a efetividade dos bancos de sangue; e sistema de saúde. ${ }^{21-23}$ Deste modo, a implementação e utilização de banco de dados e a posterior interpretação das informações obtidas possibilita definir condutas futuras, identificando e corrigindo erros, possibilitando melhor utilização de recursos, sobretudo quando se considera o aumento dos custos que complicações cirúrgicas acarretam. ${ }^{24-26}$

É preciso estar atento, no entanto, que as diferenças de gravidade entre os pacientes atendidos podem implicar diretamente em diferentes taxas de mortalidade entre hospitais ou médicos. ${ }^{15-17,27,28}$ Assim, idealmente, um ajuste de risco deve ser realizado, observando variáveis como sexo, idade, caráter de urgência da cirurgia, uso ou não de circulação extracorpórea, existência de cirurgia prévia e fração de ejeção do paciente. .11,15-17,29,30 $^{2}$

O ajuste de risco, porém, encontra dificuldades para ser realizado devido à falta ou omissão de informações importantes nos bancos de dados, sendo frequente encontrar prontuários instrumentos importantes para a criação de banco de dados - incompletos ou inadequadamente preenchidos. . $^{4,9,15,17,19,24,30}$ Logo, verifica-se a necessidade do aprimoramento dos

\section{REFERÊNCIAS}

1. Matta GC. Políticas de saúde: organização e operacionalização do Sistema Único de Saúde. Rio de Janeiro: EPSJV/ Fiocruz; 2007.

2. Brasil. Ministério da Saúde. Departamento de Informática do SUS (DATASUS). [Internet]. Brasília: Ministério da Saúde; 2008 [Acesso em: 17 maio 2019]. Disponível em: http://www2.datasus.gov.br/ DATASUS/index.php?area $=0202 \& \mathrm{id}=11633 \& \mathrm{VObj}=\mathrm{http}: / /$ tabnet . datasus.gov.br/cgi/deftohtm.exe?sih/cnv/qi

3. Piegas LS, Bittar OJ, Nogueira V, Haddad N. Cirurgia de revascularização miocárdica: resultados do Sistema Único de Saúde. Arq Bras Cardiol. 2009;93(5):555-60.

4. Bittencourt SA, Camacho LA, Leal MC. O sistema de informação hospitalar e sua aplicação na saúde coletiva Hospital Information bancos de dados para a efetivação desse meio no controle de qualidade e, assim, melhorar o desempenho das cirurgias cardiovasculares no País.

Neste trabalho, com o uso do banco de dados de cirurgia cardiovascular do HUWC, foi possível analisar o perfil das cirurgias cardiovasculares, buscando relacionar o perfil das cirurgias e a realidade na área de abrangência relacionada ao HUWC. Pela análise, foram realizadas 680 cirurgias cardiovasculares no período analisado, destacando-se o relevante papel da CRM.

É de se esperar que a CRM seja o procedimento mais frequente, devido à grande incidência de doença arterial coronariana (DAC) no Brasil, com as cirurgias orovalvares apontando em segundo lugar. ${ }^{1-3,5}$ Cabe ressaltar que o HUWC não se apresenta como o centro de referência estadual para tratamento de cardiopatias congênitas, o que pode explicar o baixo percentual de realização deste tipo de procedimento quando comparado à média nacional.

No Brasil, a maioria dos procedimentos de alta complexidade é realizada sob os auspícios do SUS. Logo, uma análise do padrão das cirurgias cardiovasculares realizadas no HUWC, e sua comparação com os dados nacionais com uso do banco de dados do DATASUS, pode refletir de forma coerente a realidade da intervenção cirúrgica cardiovascular.

\section{CONCLUSÃO}

O perfil das cirurgias cardiovasculares realizadas no HUWC ilustra que as CRM são o procedimento mais comumente realizado, seguido das cirurgias orovalvares, fato este em consonância com o verificado nacionalmente. Nota-se que as cirurgias orovalvares correspondem a um maior percentual dos procedimentos localmente, do que no restante do país, fato possivelmente atribuível à maior incidência de valvopatia reumática na região Nordeste. De posse desses dados, é possível iniciar um processo de acompanhamento que vise avaliar a qualidade dessas cirurgias, além de buscar políticas direcionadas a melhorar o serviço de oferta de cirurgias, ao mesmo tempo em que, através de práticas preventivas, diminui sua necessidade e demanda.

Systems and their application in public health. Cad Saúde Pública. 2006;22(1):19-30.

5. Agência Nacional de Saúde Suplementar. Caderno de Informações da Saúde Suplementar: beneficiários, operadoras e planos de saúde. Rio de Janeiro: ANS; 2017. 67 p.

6. Gomes WJ, Mendonça JT, Braile DM. Resultados em cirurgia cardiovascular. Oportunidade para rediscutir o atendimento médico e cardiológico no sistema público de saúde do país. Rev Bras Cir Cardiovasc. 2007;22(4):3-4.

7. Borges JB, Carvalho SM, Silva MA. Qualidade do serviço prestado aos pacientes de cirurgia cardíaca do Sistema Único de Saúde-SUS. Rev Bras Cir Cardiovasc. 2010;25(2):172-82. 
8. Braile DM, Gomes WJ. Evolução da cirurgia cardiovascular: a saga brasileira. Uma história de trabalho, pioneirismo e sucesso. Arq Bras Cardiol. 2010;94(2):151-2.

9. Murad H, Murad FF. Controle de qualidade em cirurgia cardiovascular: um paradigma a ser atingido. Rev Bras Cir Cardiovasc. 2007;22(4):470-5.

10. Ribeiro AL, Gagliardi SP, Nogueira JL, Silveira LM, Colosimo EA, Nascimento CA. Mortality related to cardiac surgery in Brazil, 2000-2003. J Thorac Cardiovasc Surg. 2006;131(4):907-9.

11. Gomes WJ, Jatene FB, Amaral JJ, Feitosa JL, Almeida RM, Cascudo MM. Registro brasileiro de cirurgias cardiovasculares em adultos. Do projeto à realidade. Rev Bras Cir Cardiovasc. 2014;29(2):III.

12. Rodés-Cabau J. Transcatheter aortic valve implantation: current and future approaches. Nat Rev Cardiol. 2012;9(1):15-29.

13. Mesquita FT, Ribeiro A, Araújo MP, Campos LA, Fernandes MA, Colafranceschi AS, et al. Indicadores de qualidade assistencial na cirurgia de revascularização miocárdica isolada em centro cardiológico terciário. Arq Bras Cardiol. 2008;90(5):350-4.

14. Lisboa LA, Moreira LF, Mejia OV, Dallan LA, Pomerantzeff PM, Costa R, et al. Evolução da cirurgia cardiovascular no Instituto do Coração: análise de 71.305 operações. Arq Bras Cardiol. 2010;94(2):174-81.

15. Edwards FH, Clark RE, Schwartz M. Practical considerations in the management of large multiinstitutional databases. Ann thorac surg. 1994;58(6):1841-4.

16. Noronha JC, Martins M, Travassos C, Campos MR, Maia P, Panezzuti R. Aplicação da mortalidade hospitalar após a realização de cirurgia de revascularização do miocárdio para monitoramento do cuidado hospitalar. Cad Saúde Pública. 2004;20(2):322-30.

17. Welke KF, Peterson ED, Vaughan-Sarrazin MS, O'Brien SM, Rosenthal GE, Shook GJ, et al. Comparison of cardiac surgery volumes and mortality rates between the Society of Thoracic Surgeons and Medicare databases from 1993 through 2001. Ann thorac surg. 2007;84(5):1538-46.

18. Ferguson TB, Dziuban SW Jr, Edwards FH, Eiken MC, Shroyer $\mathrm{AL}$, Pairolero PC, et al. The STS National Database: current changes and challenges for the new millennium. Ann thorac surg. 2000;69(3):680-91.

19. Nashef Sam, Roques F, Michel P, Gauducheau E, Lemeshow S, Salamon R, et al. European system for cardiac operative risk evaluation (EuroSCORE). Eur J Cardio Thorac Surg. 1999;16(1):9-13.
20. Codman EA. The product of a hospital. Surg Gynecol Obstret. 1914;18:491-6.

21. Miralda GP, Cuixart CB, Solé AR, Castelló PC, Peláez IM, Pons JM. Desigual perfil clínico, calidad de vida y mortalidad hospitalaria en pacientes operados de injerto aortocoronario en centros públicos y privados de Cataluña. Rev Esp Cardiol. 1998;51(10):806-15

22. Pons J, Moreno V, Borras J, Espinas J, Almazan C, Granados A. Open heart surgery in public and private practice. J Health Serv Res Policy. 1999;4(2):73-8.

23. Haddad N, Bittar E, Marchi AF, Kantorowitz CS, Ayoub AC, Fonseca ML, et al. Hospital costs of coronary artery bypass grafting on elective coronary patients. Arq Bras Cardiol. 2007;88(4):418-23.

24. Welke KF, Ferguson TB Jr, Coombs LP, Dokholyan RS, Murray CJ, Schrader MA, et al. Validity of the Society of Thoracic Surgeons National Adult Cardiac Surgery Database. Ann Thorac Surg. 2004;77(4):1137-9.

25. Grover FL, Shroyer AL, Hammermeister K, Edwards FH, Ferguson TB Jr, Dziuban SW Jr, et al. A decade's experience with quality improvement in cardiac surgery using the Veterans Affairs and Society of Thoracic Surgeons national databases. Ann Surg. 2001;234(4):464-74.

26. Arom KV, Petersen RJ, Orszulak TA, Bolman RM 3rd, Wickstrom $\mathrm{PH}$, Joyce LD, et al. Establishing and using a local/regional cardiac surgery database. Ann Thorac Surg. 1997;64(5):1245-9.

27. Landon B, Iezzoni LI, Ash AA, Shwartz M, Daley J, Hughes JS, et al. Judging hospitals by severity-adjusted mortality rates: the case of CABG surgery. Inquiry. 1996;33(2):155-66.

28. Jones RH, Hannan EL, Hammermeister KE, Delong ER, O'Connor GT, Luepker RV, et al. Identification of preoperative variables needed for risk adjustment of short-term mortality after coronary artery bypass graft surgery. The Working Group Panel on the Cooperative CABG Database Project. J Am Coll Cardiol. 1996;28(6):1478-87.

29. Bridgewater B, Neve H, Moat N, Hooper T, Jones M. Predicting operative risk for coronary artery surgery in the United Kingdom: a comparison of various risk prediction algorithms. Heart. 1998;79(4):350-5.

30. Tu JV, Sykora K, Naylor CD. Assessing the outcomes of coronary artery bypass graft surgery: how many risk factors are enough? J Am Coll Cardiol. 1997;30(5):1317-23.

\section{Como citar:}

Pimentel MD, Leite CC, Sousa CO, Bessa IL, Vieira GF, Lobo HG Filho, et al. Análise do perfil das cirurgias cardiovasculares do Hospital Universitário Walter Cantídio. Rev Med UFC. 2019 abr-jun;59(2):15-20. 